УДК 517.925.7

A. Z. Mokhonko, A. A. Mokhonko

\title{
FAST GROWING MEROMORPHIC SOLUTIONS OF THE SYSTEMS OF LINEAR DIFFERENTIAL EQUATIONS
}

\begin{abstract}
A. Z. Mokhonko, A. A. Mokhonko. Fast growing meromorphic solutions of the systems of linear differential equations, Mat. Stud. 50 (2018), 158-172.

Systems of linear differential equations that allow for dimension decrease are considered. Growth estimates for meromorphic vector-solutions are obtained. An essentially new feature is that there are no additional constraints for the growth order of the system coefficients.
\end{abstract}

Let $M$ be the field of meromorphic in $\mathbb{C}$ functions, let $\mathcal{E}$ be the ring of entire functions, $\mathcal{E} \subset M$. Consider the system

$$
\frac{d w_{j}}{d z}=\sum_{k=1}^{n} a_{j, k} w_{k}, \quad a_{j, k} \in \mathcal{E}, \quad j=1, \ldots, n .
$$

According to $[1$, Chapter $1, \S 5]$, every vector-solution $W(z)=\left(w_{1}(z), \ldots, w_{n}(z)\right), z \in \mathbb{C}$, of the system (1) has components $w_{j} \in \mathcal{E}, j=1, \ldots, n$. Applications of the Nevanlinna theory to analytic theory of differential equations are widely known, see [2]-[4]. In particular in the proof of Theorem 1 we follow the approach from [2].

Let $A$ be the coefficients matrix of the system (1):

$$
A=B_{0}(z)=\left(\begin{array}{ccccc}
s_{1} & p_{1} & 0 & \ldots & 0 \\
a_{2,1} & s_{2} & p_{2} & \ldots & 0 \\
\ldots \ldots \ldots \ldots \ldots \ldots & \ldots \ldots & \ldots \\
a_{n-1,1} & a_{n-1,2} & a_{n-1,3} & \ldots & p_{n-1} \\
a_{n, 1} & a_{n, 2} & a_{n, 3} & \ldots & s_{n}
\end{array}\right), a_{j, k}, s_{j}, p_{i} \in \mathcal{E}
$$

In [2] the properties of vector-solutions of the system (1), (2) were studied. Here the coefficients $a_{j, k}, s_{j}, p_{i}$, were entire functions of finite growth rate. In this paper a significantly new feature is that we do not pose any restrictions on the growth rate of the coefficients and solutions. The scale from [4] is used in Theorem 1 to measure an arbitrarily growth rate of positive functions.

The major idea that was used in the proof by [2] was to decrease the system dimension. This transformation leads to the system with meromorphic coefficients and meromorphic components of a vector-solution (see (42), (43)). In Theorem 2 we obtain the estimates for

2010 Mathematics Subject Classification: 30D35, 34M05, 34M10.

Keywords: system; linear differential equations; meromorphic function; entire function; Nevanlinna characteristics; order of growth; scale of growth; logarithmic derivative.

doi:10.15330/ms.50.2.158-172

(C) A. Z. Mokhonko, A. A. Mokhonko, 2018 
the growth rate of meromorphic vector-solutions for the system of linear differential equations with meromorphic coefficients.

Let us use the standard notations of the theory of meromorphic functions [6]. Landau symbols $O(\ldots), o(\ldots)$ are used in this article at $r \rightarrow+\infty$. Growth rate of $f \in M$ is described by Nevanlinna characteristics $m(r, f), T(r, f)$; remind

$$
m(r, f)=\frac{1}{2 \pi} \int_{0}^{2 \pi} \ln ^{+}\left|f\left(r e^{i \varphi}\right)\right| d \varphi, \quad \ln ^{+} x=\max (\ln x, 0), \quad x \geqslant 0 .
$$

If $f$ is an entire function then $T(r, f)=m(r, f)$. Let us denote $D(r, f)$ to be any of the characteristics $T(r, f), m(r, f)$. If $f, g \in M$, then [6, pp. 44, 45]

$$
\begin{gathered}
D(r, f+g) \leqslant D(r, f)+D(r, g)+\ln 2, \\
D(r, f \cdot g) \leqslant D(r, f)+D(r, g), \quad T\left(r, \frac{f}{g}\right) \leqslant T(r, f)+T(r, g)+O(1) .
\end{gathered}
$$

As $E$ let us denote some sets of intervals on $[0,+\infty)$ with a finite sum of lengths (mes $E<$ $+\infty)$. A function $f \in M$ has a finite growth order $\rho[f]$ if

$$
\rho=\rho[f]=\limsup _{r \rightarrow+\infty} \frac{\ln T(r, f)}{\ln r}<+\infty
$$

If $f \in M$ then the following relations are known to be true ([6, pp. 122, 125, 131])

$$
\begin{gathered}
m\left(r, \frac{f^{(k)}}{f}\right)=O(\ln r), \text { if } \rho[f]<+\infty, k=1,2, \ldots ; \\
m\left(r, \frac{f^{(k)}}{f}\right)=O\left(\ln ^{+} T(r, f)+\ln r\right), r \notin E, \text { if } \rho[f]=+\infty, k=1,2, \ldots
\end{gathered}
$$

If $F\left(f_{1}, \ldots, f_{n}\right)$ is a rational function of $f_{j} \in M, \operatorname{deg}_{f_{j}} F=k_{j}, j=1, \ldots, n$, then ([7])

$$
T\left(r, F\left(f_{1}, \ldots, f_{n}\right)\right) \leqslant \sum_{j=1, \ldots, n} k_{j} T\left(r, f_{j}\right)+O(1)
$$

if $R\left(f_{1}, \ldots, f_{n}\right)$ is a polynomial in $f_{j} \in M$, $\operatorname{deg}_{f_{j}} R=k_{j}, j=1, \ldots, n$, then

$$
m\left(r, R\left(f_{1}, \ldots, f_{n}\right)\right) \leqslant \sum_{j=1, \ldots, n} k_{j} m\left(r, f_{j}\right)+O(1)
$$

If $F(z)=\frac{P(z, f(z))}{Q(z, f(z))}=\frac{a_{1 t} f^{t}+\ldots+a_{11} f+a_{10}}{a_{2 m} f^{m}+\ldots+a_{21} f+a_{20}}$, where $f, a_{i j} \in M ; a_{1 t}, a_{2 m} \not \equiv 0 ; d=\max (m, t)$ and $P(z, w), Q(z, w)$ are relatively prime as polynomials in $w$ over the field $M$ then ([8])

$$
T(r, F)=d T(r, f)+O\left(\sum_{i, j} T\left(r, a_{i j}\right)\right)
$$

Let $W(z)=\left(w_{1}(z), \ldots, w_{n}(z)\right), w_{j} \in M, j=1, \ldots, n$. Denote

$$
T(r, W)=\max _{j=1, \ldots, n} T\left(r, w_{j}\right) .
$$


If the system (1), (2) has transcendental coefficients, then the components of its vectorsolutions $W(z)=\left(w_{1}(z), \ldots, w_{n}(z)\right)$ can be entire functions of infinite growth order $\rho\left[w_{j}\right]$ (see (4)). There are several scales for measuring growth order of the functions with the infinite growth rate. In the paper [9] for growth rate of linear differential equations solutions $p$-th iteration order $\rho_{p}(f)$ was used. In the article [10] $[p, q]$-order $\sigma_{[p, q]}(f)$ was applied. The definitions of these orders do not describe an arbitrary growth rate. This means that there exists a function $f \in \mathcal{E}$ that has an infinite $[p, q]$-rate and $p$-th iteration order for arbitrary $p \in \mathbb{N}$. There is no such a drawback in the scale proposed in [11] and adopted for various applications in [4]. As $\Phi$ let us denote the class of positive unbounded non-decreasing functions $\varphi:(0,+\infty) \rightarrow(0,+\infty)$ such that $\varphi\left(e^{t}\right)$ is slowly growing

$$
\forall c>0: \frac{\varphi\left(e^{c t}\right)}{\varphi\left(e^{t}\right)} \rightarrow 1, \quad t \rightarrow+\infty
$$

Thus if $f \in M, \varphi \in \Phi$ then the growth orders are defined as:

$$
\sigma_{\varphi}^{0}[f]=\limsup _{r \rightarrow+\infty} \frac{\varphi\left(e^{T(r, f)}\right)}{\ln r}, \quad \sigma_{\varphi}^{1}[f]=\limsup _{r \rightarrow+\infty} \frac{\varphi(T(r, f))}{\ln r} .
$$

From (11) it follows $\forall c>0: \varphi\left(\left(e^{t}\right)^{c}\right)=(1+o(1)) \varphi\left(e^{t}\right), t \rightarrow+\infty$; if we denote $x=e^{t}$ then the previous iimplies

$$
\forall \varphi \in \Phi \quad \forall c>0: \quad \varphi\left(x^{c}\right)=(1+o(1)) \varphi(x), \quad x>x_{0}
$$

For the functions $\varphi \in \Phi$ it holds ([4])

$$
\forall \varphi \in \Phi \quad \forall m>0 \quad \forall k \geqslant 0: \quad \frac{\varphi^{-1}\left(\ln x^{m}\right)}{x^{k}} \rightarrow+\infty .
$$

In particular, $\forall \varphi \in \Phi \forall m>0: \quad x<\varphi^{-1}\left(\ln x^{m}\right), \quad x>x_{0}$. Thus

$$
\forall \varphi \in \Phi \forall m>0: \ln x<\ln \varphi^{-1}\left(\ln x^{m}\right), \quad x>x_{0} .
$$

Due to the result of Filevych ([12]) we have:

$$
(\forall f \in \mathcal{E}, \rho[f]=+\infty)(\exists \varphi \in \Phi): \quad \sigma_{\varphi}^{0}[f]=1 .
$$

This means that the function $f$ has a finite positive growth order $\sigma_{\varphi}^{0}[f]$. This statement allows estimating the growth order of vector-solutions of the fundamental system of solutions of (1), (2) via the growth order of its coefficients.

If $\sigma_{\varphi}^{1}[f]=\sigma<+\infty$ then taking into account (12) we have $\forall \varepsilon>0: \varphi(T(r, f))<$ $\ln r^{\sigma+\varepsilon}, r>r_{0}$. Then

$$
\sigma_{\varphi}^{1}[f]=\sigma \Rightarrow T(r, f)<\varphi^{-1}\left(\ln r^{\sigma+\varepsilon}\right), \quad \varepsilon>0, \quad r>r_{0}
$$

If $g \in M$ and $\sigma_{\varphi}^{0}[g]=\alpha<+\infty$ then by taking into account (12) we obtain $\forall \varepsilon>0$ : $\varphi\left(e^{T(r, g)}\right)<\ln r^{\alpha+\varepsilon}, \quad r>r_{0}$. Thus

$$
\sigma_{\varphi}^{0}[g]=\alpha \Rightarrow T(r, g)<\ln \varphi^{-1}\left(\ln r^{\alpha+\varepsilon}\right), \quad \varepsilon>0, \quad r>r_{0} .
$$


Denote, see $(2)(j=1, \ldots, n ; t=1, \ldots, n-j+1)$

$$
\begin{aligned}
& d_{j t}(A)=\left|\begin{array}{ccccc}
s_{t} & p_{t} & 0 & \ldots & 0 \\
a_{t+1, t} & s_{t+1} & p_{t+1} & \ldots & 0 \\
\ldots \ldots \ldots \ldots \ldots \ldots \ldots \ldots \ldots \ldots \ldots & \ldots \ldots \ldots \\
a_{t+j-2, t} & a_{t+j-2, t+1} & a_{t+j-2, t+2} & \ldots & p_{t+j-2} \\
a_{t+j-1, t} & a_{t+j-1, t+1} & a_{t+j-1, t+2} & \ldots & s_{t+j-1}
\end{array}\right|, \\
& d_{0, t} \equiv 1, \quad H_{j}(A)=\sum_{t=1}^{n+1-j} d_{j, t}(A) .
\end{aligned}
$$

The main result of this article is the following

Theorem 1. Let the system (1), (2) be such that all coefficients $a_{j, k}, s_{j}, p_{i} \in \mathcal{E}$, and $m \in$ $\{0,1, \cdots, n-1\}$

$$
\sigma_{\varphi}^{0}\left[H_{n-m}(A)\right]>\sigma_{\varphi}^{0}\left[d_{j t}(A)\right], \quad j=1,2, \ldots, n-m-1 ; \quad t=1, \ldots, n-j+1 .
$$

Then there exist no $m+1$ linear independent meromorphic vector-solutions $W_{k}(z)=\left(w_{k 1}(z)\right.$, $\left.\ldots, w_{k n}(z)\right)$, of the system (1), (2) such that

$$
\sigma_{\varphi}^{1}\left[W_{k}\right]<\sigma_{\varphi}^{0}\left[H_{n-m}(A)\right], \quad k=0,1, \ldots, m .
$$

The following Theorem 2 is similar to Theorem 1, though they do not follow one from another. If in the system (1), (2) the coefficients $a_{j, k}, s_{j}, p_{i} \in M$ and $P$ is the set of poles of all coefficients, then according to [1, Chapter $1, \S 5]$ every vector-solution has components, that are analytic functions in $\mathbb{C} \backslash P$. We are interested in vector-solutions $W(z)=\left(w_{1}(z), \ldots\right.$, $\left.w_{n}(z)\right)$ with components $w_{j} \in M, j=1, \ldots, n$.

Theorem 2. Let the system (1), (2) be such that all coefficients $a_{j, k}, s_{j}, p_{i} \in M$, and $(m \in\{0,1, \cdots, n-1\}, j=1,2, \cdots, n-m-1)$

$$
m\left(r, d_{j t}(A)\right)=o\left(m\left(r, H_{n-m}(A)\right)\right), \quad r \notin E ; \quad t=1, \ldots, n-j+1 .
$$

Then there exists no $m+1$ linear independent meromorphic vector-solutions $W_{k}(z)=$ $\left(w_{k 1}(z), \ldots, w_{k n}(z)\right), \quad k=0,1, \ldots, m$, of the system (1), (2) such that $\ln \left(r \cdot T\left(r, W_{k}\right)\right)=$ $o\left(m\left(r, H_{n-m}(A)\right)\right), \quad r \notin E$; (whose growth rate is restricted by growth rate of the coefficients).

Remark 1. If we apply more precise estimates of logarithmic derivative (5) for important sub-classes of meromorphic functions then the following can be obtained: if the coefficients of the system (1), (2) are such that

$$
\begin{gathered}
m\left(r, d_{j t}(A)\right)=O(\ln r), \quad j=1,2, \ldots, n-m-1 ; \quad t=1, \ldots, n-j+1 \\
m\left(r, H_{n-m}(A)\right) \neq O(\ln r)
\end{gathered}
$$

then the system has no more than $m$ linearly-independent meromorphic vector-solutions $W_{k}, k=1,2, \ldots, m$ of finite growth order. The relations (23) hold true if e.g. $d_{j t}(A)$ are any rational functions and $H_{n-m}(A)$ is transcendent function. In fact, a transcendent function grows faster than any rational function [6, pp. 49, 50]. 
Example 1. Consider the system $w_{1}^{\prime}=w_{2}, w_{2}^{\prime}=e^{2 z} w_{1}+w_{2}$. The matrix of the system is $A=\left(\begin{array}{cc}0 & 1 \\ \exp 2 z & 1\end{array}\right), d_{11}(A)=0, d_{12}=1 ; H_{2}(A)=-e^{2 z}, m\left(r, H_{2}(A)\right)=2 m\left(r, e^{z}\right)=\frac{2 r}{\pi}$ ([13, p. 25]). We have: $0=m\left(r, d_{11}(A)\right)=m\left(r, d_{12}(A)\right)=o\left(m\left(r, H_{2-0}(A)\right)\right)$. In this example $n=2$, $m=0$. Thus from Theorem 2 it follows that the system does not have meromorphic vectorsolutions $W$ such that $\ln ^{+} T(r, W)+\ln r=o\left(m\left(r, H_{2-0}(A)\right)\right), r \notin E$. This system has two linearly-independent meromorphic vector-solutions $W_{1}=\left(e^{e^{z}}, e^{z} e^{e^{z}}\right), W_{2}=\left(e^{-e^{z}},-e^{z} e^{-e^{z}}\right)$. For entire function $\exp \exp z\left([13\right.$, p. 26] $) T\left(r, e^{e^{z}}\right)=m\left(r, e^{e^{z}}\right) \sim \frac{e^{r}}{\left(2 \pi^{3} r\right)^{1 / 2}}, r \rightarrow+\infty$. Taking into account (9) it follows $T\left(r, e^{z} e^{-e^{z}}\right)=T\left(r, e^{e^{z}}\right)+O\left(T\left(r, e^{z}\right)\right) \sim \frac{e^{r}}{\left(2 \pi^{3} r\right)^{1 / 2}}, r \rightarrow+\infty$. Thus keeping in mind the definition $W_{1}, W_{2}$, we obtain $T\left(r, W_{j}\right) \sim \frac{e^{r}}{\left(2 \pi^{3} r\right)^{1 / 2}}, r \rightarrow+\infty, j=1,2$. Thus $r \sim \ln \left(r \cdot T\left(r, W_{j}\right)\right) \neq o\left(m\left(r, H_{2}(A)\right)\right), r \rightarrow+\infty$ because $m\left(r, H_{2}(A)\right) \sim \frac{2 r}{\pi}, r \rightarrow+\infty$.

Example 2. The system $w_{1}^{\prime}=w_{2}, w_{2}^{\prime}=w_{2}\left(1+e^{z}\right)$ has the matrix $A=\left(\begin{array}{cc}0 & 1 \\ 0 & 1+e^{z}\end{array}\right) ; H_{1}=$ $H_{2-1}(A)=1+e^{z} ; n=2, m=1 ; \quad m\left(r, H_{1}(A)\right)=m\left(r, e^{z}+1\right)=m\left(r, e^{z}\right)+O(1) \sim \frac{r}{\pi}$, $r \rightarrow+\infty$. A fundamental system consists of two linearly independent meromorphic vectorsolutions. According to Theorem 2 this fundamental system has no more than one meromorphic vector-solution $W$ such that $\ln ^{+} T(r, W)+\ln r=o\left(m\left(r, H_{1}(A)\right)\right), r \notin E$. This solution is $W_{1}=(1,0)$. The second linearly independent solution of the fundmental system is $W_{2}=\left(e^{e^{z}}, e^{z} e^{e^{z}}\right)$. Similar to Example $1 T\left(r, W_{2}\right) \sim \frac{e^{r}}{\left(2 \pi^{3} r\right)^{1 / 2}}, \ln ^{+} T\left(r, W_{2}\right)+\ln r \sim r, r \rightarrow$ $+\infty$. Thus $r \sim \ln \left(r \cdot T\left(r, W_{2}\right)\right) \neq o\left(m\left(r, H_{1}(A)\right)\right), r \rightarrow+\infty$, because $m\left(r, H_{1}(A)\right) \sim \frac{r}{\pi}$, $r \rightarrow+\infty$.

Let us consider the vector $h(z)=\left(h_{1}, h_{2}, \ldots, h_{n}\right)$ where $h_{j} \in M$. Denote

$$
Q_{0}(A, h) \equiv 1, \quad Q_{k}(A, h)=\left|\begin{array}{ccccc}
s_{1}-h_{1} & p_{1} & 0 & \ldots & 0 \\
a_{2,1} & s_{2}-h_{2} & p_{2} & \ldots & 0 \\
\ldots \ldots \ldots \ldots \ldots \ldots & \ldots & \ldots & \ldots \\
a_{k-1,1} & a_{k-1,2} & a_{k-1,3} & \ldots & p_{k-1} \\
a_{k, 1} & a_{k, 2} & a_{k, 3} & \ldots & s_{k}-h_{k}
\end{array}\right|,
$$

$k=1,2, \ldots, n$. By using $(24)$ we have $\left(d_{1, k}=s_{k}\right)$

$$
\begin{aligned}
& Q_{k}=-h_{k} Q_{k-1}+\left|\begin{array}{cccccc}
s_{1}-h_{1} & p_{1} & 0 & \ldots & 0 & 0 \\
a_{2,1} & s_{2}-h_{2} & p_{2} & \ldots & 0 & 0 \\
\ldots \ldots \ldots \ldots \ldots \ldots \ldots \ldots \ldots & \ldots & \ldots \ldots \ldots \ldots \\
a_{k-1,1} & a_{k-1,2} & a_{k-1,3} & \ldots & s_{k-1}-h_{k-1} & p_{k-1} \\
a_{k, 1} & a_{k, 2} & a_{k, 3} & \ldots & a_{k, k-1} & s_{k}
\end{array}\right|= \\
& =-h_{k} Q_{k-1}-Q_{k-2} h_{k-1} d_{1, k}+\left|\begin{array}{cccccc}
s_{1}-h_{1} & p_{1} & 0 & \ldots & 0 & 0 \\
a_{2,1} & s_{2}-h_{2} & p_{2} & \ldots & 0 & 0 \\
\ldots \ldots \ldots \ldots \ldots \ldots & \ldots & \ldots & \ldots & \ldots \\
a_{k-1,1} & a_{k-1,2} & a_{k-1,3} & \ldots & s_{k-1} & p_{k-1} \\
a_{k, 1} & a_{k, 2} & a_{k, 3} & \ldots & a_{k, k-1} & s_{k}
\end{array}\right|= \\
& =\ldots=d_{k, 1}(A)-\sum_{i=0}^{k-1} Q_{i}(A, h) h_{i+1} d_{k-i-1, i+2}(A), \quad d_{0, k+1}(A)=1 .
\end{aligned}
$$

Lemma 1. The determinant $Q_{k}(A, h)$ can be represented as

$$
Q_{k}(A, h)=d_{k 1}(A)-d_{k-1,1}(A) h_{k}+\sum_{j=0}^{k-2} d_{j 1}(A) P_{j k}, \quad k=1,2, \ldots, n,
$$


where $h=\left(h_{1}, h_{2} \ldots, h_{n}\right) ; \quad P_{j k}$ is a polynomial in functions $h_{t}$ and $d_{\nu s}(A), j+1 \leqslant t \leqslant k$, $j+2 \leqslant s \leqslant k, \nu<k$, of degree at most 1 for every $h_{t}, d_{\nu s}$.

Proof of Lemma 1. Taking into account the definitions $(24)$, (18) we have $\left(d_{01}=1\right)$ $Q_{1}(A, h)=s_{1}-h_{1}=d_{11}-d_{01} h_{1}, Q_{2}(A, h)=d_{21}-d_{11} h_{2}-d_{01}\left(d_{12} h_{1}-h_{1} h_{2}\right)=d_{21}-$ $d_{11} h_{2}-d_{01} P_{02}, \quad Q_{3}(A, h)=d_{31}-d_{22} h_{1}-h_{2} Q_{1}(A, h) d_{13}-h_{3} Q_{2}(A, h)=d_{31}-d_{21} h_{3}+$ $d_{11}\left(h_{2} h_{3}-h_{2} d_{13}\right)+d_{01}\left(d_{13} h_{1} h_{2}-h_{1} d_{22}+d_{12} h_{1} h_{3}-h_{1} h_{2} h_{3}\right)=d_{31}-d_{21} h_{3}+d_{11} P_{13}+d_{01} P_{03}$. The assumptions of the lemma preconditions for the polynomials $P_{02}, P_{13}, P_{03}$ hold true.

Assume that the statement of the Lemma are proved for all $Q_{i}, i=1, \ldots, k-1$. Let us prove it for $Q_{k}, k \geqslant 4$. By substituting into (25) the decompositions $Q_{i}$ of the form (26), after simple transformation we obtain $\left(d_{0, k+1}=1\right)(k \geqslant 4)$

$$
\begin{gathered}
Q_{k}=d_{k 1}-h_{k}\left(d_{k-1,1}-d_{k-2,1} h_{k-1}+\sum_{j=0}^{k-3} d_{j 1} P_{j, k-1}\right)-Q_{1} h_{2} d_{k-2,3}-Q_{0} h_{1} d_{k-1,2}- \\
-\sum_{i=2}^{k-2}\left(d_{i 1}-d_{i-1,1} h_{i}+\sum_{j=0}^{i-2} d_{j 1} P_{j, i}\right) h_{i+1} d_{k-i-1, i+2}=d_{k 1}-h_{k} d_{k-1,1}- \\
-Q_{1} h_{2} d_{k-2,3}-Q_{0} h_{1} d_{k-1,2}-\sum_{i=2}^{k-2} \sum_{j=0}^{i-2} d_{j 1} P_{j, i} h_{i+1} d_{k-i-1, i+2}-\sum_{1}-\sum_{2}+\sum_{3} ; \\
\sum_{1}=\sum_{j=0}^{k-3} d_{j 1} P_{j, k-1} h_{k}-d_{k-2,1} h_{k-1} h_{k} \stackrel{\text { def }}{=} \sum_{j=0}^{k-2} d_{j 1} P_{j, k}^{1} ; \\
\sum_{2}=\sum_{i=2}^{k-2} d_{i 1} h_{i+1} d_{k-i-1, i+2} \stackrel{\text { def }}{=} \sum_{i=2}^{k-2} d_{i 1} P_{i, k}^{2} \\
\sum_{3}=\sum_{i=2}^{k-2} d_{i-1,1} h_{i} h_{i+1} d_{k-i-1, i+2} \stackrel{\text { def }}{=} \sum_{i=2}^{k-2} d_{i-1,1} P_{i-1, k}^{3} ; \\
Q_{1} h_{2} d_{k-2,3}=d_{11} h_{2} d_{k-2,3}-d_{01} h_{1} h_{2} d_{k-2,3} \stackrel{\text { def }}{=} d_{11} P_{1, k}^{4}+d_{01} P_{0, k}^{4} \\
Q_{0} h_{1} d_{k-1,2}=d_{01} h_{1} d_{k-1,2} \stackrel{\text { def }}{=} d_{01} P_{0, k}^{5}, d_{01}=1, \quad Q_{0}=1 ; \\
\sum_{i=2}^{k-2} \sum_{j=0}^{i-2} d_{j 1} P_{j, i} h_{i+1} d_{k-i-1, i+2}=\sum_{j=0}^{k-4} d_{j 1} \sum_{i=j+2}^{k-2} P_{j, i} h_{i+1} d_{k-i-1, i+2} .
\end{gathered}
$$

From induction hypothesis about polynomial properties $P_{j, k-1}$ and the definitions of the polynomials $P_{j, k}^{s}, s=1,2, \ldots, 5, j=0,1, \ldots, k-2$, it follows that $P_{j, k}^{s}$ are some polynomials in $h_{t}$ and $d_{\nu s}, \quad j+1 \leqslant t \leqslant k, \quad j+2 \leqslant t \leqslant k, \quad \nu<k$ of degree no more than 1 in every $h_{t}, d_{\nu s}$. By grouping the summands that contain $d_{j 1}, j=0,1, \ldots, k-2$ we obtain

$$
\sum_{1}+\sum_{2}-\sum_{3}-Q_{1} h_{2} d_{k-2,3}-Q_{0} h_{1} d_{k-2,2} \stackrel{\text { def }}{=} \sum_{j=0}^{k-2} d_{j 1} P_{j, k}^{*}
$$

where $P_{j, k}^{*}$ are some polynomials in $h_{t}$ and $d_{\nu s}, \quad j+1 \leqslant t \leqslant k, \quad j+2 \leqslant t \leqslant k, \quad \nu<k$ of degree no more than 1 on every $h_{t}, d_{\nu s}$. After substitution of (27), (28) into the expression for $Q_{k}$ and grouping the summands that contain $d_{j 1}, j=0,1, \ldots, k-2$ we obtain (26).

Proof of Theorem 1. The case $m=n-1$ will be considered in a more general form. Consider the system (1) with the coefficients $a_{k j} \in \mathcal{E}$ (condition (2) may not be satisfied). Let $\operatorname{Sp} A=$ $a_{11}+a_{22}+\ldots+a_{n n}=H_{1}(A)$ be the trace of matrix $A$ of the system (1). Let us prove that the system (1) does not have $m+1=n$ linearly independent vector-solutions $W_{k}=$ $\left(w_{k 1}, \ldots, w_{k n}\right)$ such that

$$
\sigma_{\varphi}^{0}[\operatorname{Sp} A]>\sigma_{\varphi}^{1}\left[W_{k}\right] \stackrel{\text { def }}{=} \sigma_{k}, \quad k=1, \ldots, n .
$$


Assume that there exist $n$ linearly independent vector-solutions $W_{k}$ for which (29) holds true. Then $W_{k}, k=1, \ldots, n$, is the fundamental system of solutions for (1) with the determinant $D(z)$ satisfying the equality $\frac{D^{\prime}(z)}{D(z)}=\operatorname{Sp} A$. Then

$$
m(r, \operatorname{Sp} A)=m\left(r, \frac{D^{\prime}(z)}{D(z)}\right) \stackrel{(6)}{=} O\left(\ln ^{+} T(r, D)+\ln r\right), \quad r \notin E .
$$

Taking into account the definition of $D(z)$ and the estimate (7) we obtain:

$$
T(r, D) \stackrel{(10)}{\leqslant} \sum_{1 \leqslant k, j \leqslant n} T\left(r, w_{k, j}\right)+O(1) \leqslant n^{2} \max _{k=1, \ldots, n} T\left(r, W_{k}\right)+O(1) .
$$

From (29), (16) we have $\varphi\left(T\left(r, W_{k}\right)\right)<\ln r^{\sigma_{k}+\varepsilon}, \varepsilon>0$. Let $\sigma=\max \sigma_{k}, k=1, \ldots, n$. Then $T\left(r, W_{k}\right)<\varphi^{-1}\left(\ln r^{\sigma+\varepsilon}\right), \quad \varepsilon>0, \quad k=1, \ldots, n$, and by taking into account (31), $T(r, D)=O\left(\varphi^{-1}\left(\ln r^{\sigma+\varepsilon}\right)\right), \quad \varepsilon>0$. Then from (30) it follows $(K=$ const $>0)$

$$
m(r, \operatorname{Sp} A)<K\left(\ln ^{+} T(r, D)+\ln r\right) \stackrel{(14)}{<} 2 K\left(\ln \varphi^{-1}\left(\ln r^{\sigma+\varepsilon}\right)\right), r \notin E .
$$

If $r>$ mes $E$ then $\exists r_{1} \in[r, 2 r] \backslash E$. Since the function $\operatorname{Sp} A$ is entire then the functions $m(r, \operatorname{Sp} A), \ln \varphi^{-1}\left(\ln r^{\sigma+\varepsilon}\right)$ are increasing. So we have:

$$
\begin{gathered}
m(r, \operatorname{Sp} A) \leqslant m\left(r_{1}, \operatorname{Sp} A\right)<2 K\left(\ln \varphi^{-1}\left(\ln r_{1}^{\sigma+\varepsilon}\right)\right) \leqslant \\
\leqslant 2 K\left(\ln \varphi^{-1}\left(\ln (2 r)^{\sigma+\varepsilon}\right)\right)<2 K\left(\ln \varphi^{-1}\left(\ln r^{\sigma+2 \varepsilon}\right)\right), \quad r>r_{0}, \quad \varepsilon>0 .
\end{gathered}
$$

Therefore $m(r, \operatorname{Sp} A)=O\left(\ln \varphi^{-1}\left(r^{\sigma+2 \varepsilon}\right)\right), \quad r>r_{0}, \quad \varepsilon>0$. So

$$
\begin{gathered}
\varphi\left(e^{m(r, \operatorname{Sp} A)}\right)=\varphi\left(e^{O\left(\ln \varphi^{-1}\left(\ln r^{\sigma+2 \varepsilon}\right)\right)}\right) \stackrel{(13)}{<}(1+o(1)) \varphi\left(e^{\ln \varphi^{-1}\left(\ln r^{\sigma+2 \varepsilon}\right)}\right)= \\
=(1+o(1)) \ln r^{\sigma+2 \varepsilon}, \quad r>r_{0} .
\end{gathered}
$$

By taking into account the definition $\sigma_{\varphi}^{0}[\operatorname{Sp} A]$ and the fact that for the entire function $m(r, \operatorname{Sp} A)=T(r, \operatorname{Sp} A)$ we obtain $\sigma_{\varphi}^{0}[\operatorname{Sp} A] \leqslant \sigma+2 \varepsilon$. Thus $\sigma_{\varphi}^{0}[\operatorname{Sp} A] \leqslant \sigma=\max \sigma_{k}, k=$ $1, \ldots, n$, which contradicts $(29)$.

Suppose now that in $(2)$ all $p_{j} \not \equiv 0, j=1, \ldots, n-1$. If $W=\left(w_{1}, \ldots, w_{n}\right)$ is a non-trivial meromorphic vector-solution of the system (1), (2) then from matrix (2) structure it follows that $w_{1} \not \equiv 0$.

Let $m=0$. Then $n-m=n$,

$$
H_{n-m}(A)=H_{n}(A)=d_{n 1}(A) .
$$

Assume that there exists a non-trivial meromorphic solution $W=\left(w_{1}, \ldots, w_{n}\right)$ of the system (1), (2) such that

$$
\sigma \stackrel{\text { def }}{=} \sigma_{\varphi}^{1}[W]<\sigma_{\varphi}^{0}\left[H_{n}(A)\right] \stackrel{\text { def }}{=} \alpha .
$$

Let us rewrite the system (1), (2) as:

$$
\begin{aligned}
& w_{1}\left(s_{1}-w_{1}^{\prime} / w_{1}\right)+p_{1} w_{2}=0, \\
& w_{1} a_{21}+w_{2}\left(s_{2}-w_{2}^{\prime} / w_{2}\right)+w_{3} p_{2}=0, \\
& \ldots \ldots \ldots \ldots \ldots \ldots \ldots \ldots \ldots \ldots \ldots \ldots \ldots \ldots \ldots \ldots \ldots \\
& w_{1} a_{n 1}+\ldots+w_{n-1} a_{n, n-1}+w_{n}\left(s_{n}-w_{n}^{\prime} / w_{n}\right)=0 .
\end{aligned}
$$


This system has a non-trivial solution $W(z)$. So $(24) Q_{n}\left(A, h_{0}\right) \equiv 0$, where $h_{0}=\left(h_{01}, \ldots, h_{0 n}\right)$, $h_{0 j}=w_{j}^{\prime} / w_{j}, j=1, \ldots, n$. From Lemma 1 it follows

$$
0 \equiv Q_{n}\left(A, h_{0}\right) \stackrel{(26)}{=} d_{n 1}(A)-d_{n-1,1}(A) h_{0 n}+\sum_{j=0}^{n-2} d_{j 1}(A) P_{j n}
$$

where $P_{j n}$ are some polynomials in functions $h_{0 t}=w_{t}^{\prime} / w_{t}, j+1 \leqslant t \leqslant n$, and $d_{\nu s}(A), \nu<$ $n, j+2 \leqslant s \leqslant n$, of degree no more than 1 in every of $h_{0 t}$, and $d_{\nu s}$. Thus taking into account (34) we have

$$
H_{n}(A)=d_{n-1,1}(A) h_{0 n}-\sum_{j=0}^{n-2} d_{j 1}(A) P_{j n} .
$$

From this equality and properties of the polynomials $P_{j n}$ it follows:

$$
\begin{aligned}
& m\left(r, H_{n}(A)\right) \stackrel{(8)}{\leqslant} \sum_{j=1}^{n} m\left(r, \frac{w_{j}^{\prime}}{w_{j}}\right)+\sum_{\substack{1 \leqslant j \leqslant n-1, 1 \leqslant t \leqslant n-j+1}} m\left(r, d_{j t}\right)+O(1) \stackrel{(6)}{\leqslant} \\
& \leqslant O\left(\sum_{j=1}^{n} \ln ^{+} T\left(r, w_{j}\right)+\ln r\right)+\sum_{\substack{1 \leqslant j \leqslant n-1, 1 \leqslant t \leqslant n-j+1}} m\left(r, d_{j t}\right), \quad r \notin E .
\end{aligned}
$$

Inequality (20) for $m=0$ implies

$$
\beta_{j t} \stackrel{\text { def }}{=} \sigma_{\varphi}^{0}\left[d_{j t}\right]<\sigma_{\varphi}^{0}\left[H_{n}(A)\right] \stackrel{\text { def }}{=} \alpha, j=1, \ldots, n-1 ; t=1, \ldots, n-j+1 .
$$

Let us denote $\max \beta_{j t} \stackrel{\text { def }}{=} \beta \stackrel{(39)}{<} \alpha$. For the entire function $d_{j t}(A)$ the following equality $T\left(r, d_{j t}\right)=m\left(r, d_{j t}\right)$ holds. Keeping in mind (17), (39) this gives us:

$$
m\left(r, d_{j t}\right)<\ln \varphi^{-1}\left(\ln r^{\beta+\varepsilon}\right), \quad \varepsilon>0 .
$$

From (10), (35) it follows

$$
T\left(r, w_{j}\right) \stackrel{(10)}{\leqslant} T(r, W) \stackrel{(35),(16)}{<} \varphi^{-1}\left(\ln r^{\sigma+\varepsilon}\right), \quad \varepsilon>0, \quad r>r_{0}
$$

From (38), (41), (14), (40) we obtain $(K=$ const $>0) m\left(r, H_{n}(A)\right)<K \cdot\left(\ln \varphi^{-1}\left(\ln r^{\sigma+\varepsilon}\right)+\right.$ $\left.+\ln \varphi^{-1}\left(\ln r^{\beta+\varepsilon}\right)\right)<2 K \ln \varphi^{-1}\left(\ln r^{\max (\sigma, \beta)+\varepsilon}\right), \quad r \notin E$. Similar to (33) we obtain:

$$
\begin{gathered}
m\left(r, H_{n}(A)\right)=O\left(\ln \varphi^{-1}\left(\ln r^{\max (\sigma, \beta)+2 \varepsilon}\right)\right), \quad r>r_{0}, \quad \varepsilon>0 ; \\
e^{m\left(r, H_{n}(A)\right)}=e^{O\left(\ln \varphi^{-1}\left(\ln r^{\max (\sigma, \beta)+2 \varepsilon}\right)\right)} ; \quad \varphi\left(e^{m\left(r, H_{n}(A)\right)}\right)=\varphi\left(e^{O\left(\ln \varphi^{-1}\left(\ln r^{\max (\sigma, \beta)+2 \varepsilon}\right)\right)}\right) \stackrel{(13)}{<} \\
<(1+o(1)) \varphi\left(e^{\ln \varphi^{-1}\left(\ln r^{\max (\sigma, \beta)+2 \varepsilon}\right.}\right)=(1+o(1)) \ln r^{\max (\sigma, \beta)+2 \varepsilon}, \quad r>r_{0} .
\end{gathered}
$$

From this estimate, from (12) and from the fact that for entire function $m\left(r, H_{n}(A)\right)=$ $T\left(r, H_{n}(A)\right)$ we conclude that $\sigma_{\varphi}^{0}\left[H_{n}(A)\right] \leqslant \max (\sigma, \beta)$, which contradicts (35), (39).

Let $0<m<n-1$. Suppose that there exists $m+1$ linearly-independent meromorphic vector-solutions $W_{k}=\left(w_{k 1}, \ldots, w_{k n}\right), k=0, \ldots, m$ of the system (1), (2) such that (21) holds. One of these $m+1$ solutions e.g. $W_{0}$ we denote by $U, W_{0}=U=\left(u_{1}, \ldots, u_{n}\right)=$ $\left(w_{01}, \ldots, w_{0 n}\right)$. Any of the remaining $m$ meromorphic vector-solutions is denoted by $W=$ 
$\left(w_{1}, \ldots, w_{n}\right)$. Since $U$ is a non-trivial meromorphic vector-solution of the system $(1),(2)$ then $u_{1} \not \equiv 0$. Let us describe the transformation from the system (1) with coefficient matrix (2) of the dimension $n$ to the system of differential equations with a coefficient matrix of the form (2) and dimension $n-1$.

For every of $m$ meromorphic vector-solutions $W=\left(w_{1}, \ldots, w_{n}\right)$ of the system (1), (2) let us assign the corresponding vector

$$
V=\left(v_{1}, v_{2}, \ldots, v_{n}\right)=\left(\frac{w_{1}}{u_{1}}, w_{2}-\frac{w_{1} u_{2}}{u_{1}}, \ldots, w_{n}-\frac{w_{1} u_{n}}{u_{1}}\right), v_{1}=\frac{w_{1}}{u_{1}} \not \equiv 0 .
$$

From (1), (2), (42) it follows that these $m$ vectors $V(42)$ are the solutions of the system $[2$, formulae $(3,9)-(3,13)]$

$$
\begin{aligned}
& v_{1}^{\prime}=v_{2} p_{1} / u_{1} \\
& v_{2}^{\prime}=v_{2}\left(s_{2}-p_{1} u_{2} / u_{1}\right)+p_{2} v_{3} \\
& \ldots \ldots \ldots \ldots \ldots \ldots \ldots \ldots \ldots \ldots \ldots \ldots \ldots \ldots \ldots \ldots \ldots \ldots \ldots \ldots \ldots \\
& v_{n}^{\prime}=v_{2}\left(a_{n 2}-p_{1} u_{n} / u_{1}\right)+\sum_{k=3}^{n-1} a_{n k} v_{k}+s_{n} v_{n}
\end{aligned}
$$

whose coefficients matrix has the form

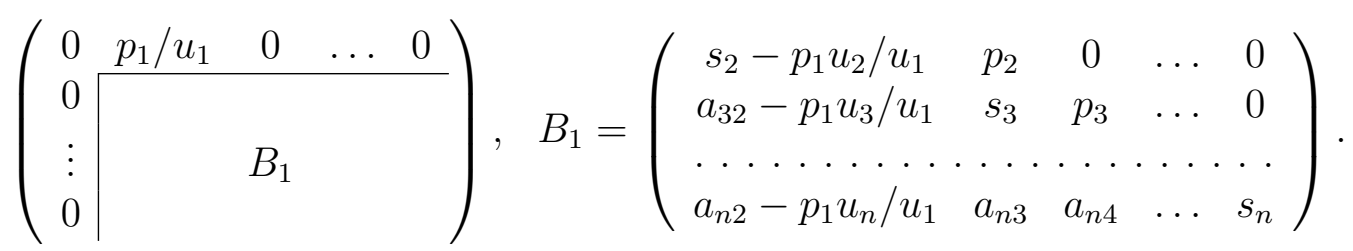

Lemma 2. The following relations hold true $\left(d_{0, j+2}(A)=1\right)$

$$
\begin{gathered}
d_{j, k-1}\left(B_{1}\right)=d_{j k}(A), k>2, \quad 1 \leqslant j \leqslant n-2, \\
d_{j 1}\left(B_{1}\right)=d_{j 2}(A)+\sum_{k=1}^{j} Q_{k}(A, h) d_{j-k, k+2}(A), \quad j=1,2, \ldots, n-1,
\end{gathered}
$$

where $h=h_{0}=\left(u_{1}^{\prime} / u_{1}, \ldots, u_{n}^{\prime} / u_{n}\right)=\left(w_{01}^{\prime} / w_{01}, \ldots, w_{0 n}^{\prime} / w_{0 n}\right)$.

Proof of Lemma 2. If $k>2$, then the first of equations (45) follows from the definition of $d_{j, k-1}\left(B_{1}\right)$ and matrix $B_{1}(44)$. If $k=2$ then

$$
\begin{aligned}
& d_{j, 1}\left(B_{1}\right) \stackrel{(44)}{=} d_{j 2}(A)-\frac{p_{1}}{u_{1}}\left|\begin{array}{ccccc}
u_{2} & p_{2} & 0 & \ldots & 0 \\
u_{3} & s_{3} & p_{3} & \ldots & 0 \\
\ldots \ldots & \ldots & \ldots & \ldots & \ldots \\
u_{j} & a_{j, 3} & a_{j, 4} & \ldots & p_{j} \\
u_{j+1} & a_{j+1,3} & a_{j+1,4} & \ldots & s_{j+1}
\end{array}\right|=d_{j 2}(A)- \\
& -\frac{p_{1} u_{2}}{u_{1}} d_{j-1,3}+\frac{p_{1} p_{2}}{u_{1}}\left|\begin{array}{ccccc}
u_{3} & p_{3} & 0 & \ldots & 0 \\
u_{4} & s_{4} & p_{4} & \ldots & 0 \\
\ldots \ldots & \ldots & \ldots & \ldots & \ldots \\
u_{j} & a_{j, 4} & a_{j, 5} & \ldots & p_{j} \\
u_{j+1} & a_{j+1,4} & a_{j+1,5} & \ldots & s_{j+1}
\end{array}\right|=d_{j 2}-\frac{p_{1} u_{2}}{u_{1}} \times
\end{aligned}
$$




$$
\begin{gathered}
\times d_{j-1,3}+\frac{p_{1} p_{2}}{u_{1}} u_{3} d_{j-2,4}(A)-\frac{p_{1} p_{2}}{u_{1}} p_{3}\left|\begin{array}{ccccc}
u_{4} & p_{4} & 0 & \ldots & 0 \\
u_{5} & s_{5} & p_{5} & \ldots & 0 \\
\ldots & \ldots & \ldots & \ldots & \ldots \\
u_{j} & a_{j, 5} & a_{j, 6} & \ldots & p_{j} \\
u_{j+1} & a_{j+1,5} & a_{j+1,6} & \ldots & s_{j+1}
\end{array}\right|= \\
=d_{j 2}(A)+\sum_{k=1}^{j}(-1)^{k} \frac{u_{k+1}}{u_{1}} p_{1} p_{2} \ldots p_{k} d_{j-k, k+2}(A) .
\end{gathered}
$$

It is known [2] that $(-1)^{k} \frac{u_{k+1}}{u_{1}} p_{1} p_{2} \ldots p_{k}=Q_{k}(A, h), h=\left(u_{1}^{\prime} / u_{1}, \ldots, u_{n}^{\prime} / u_{n}\right)$. So we obtain (45).

The matrix $B_{1}$ (44) has the form (2). Taking into account (43), (42) each of $m$ vectors

$$
Y_{1}=\left(v_{2}, v_{3} \ldots, v_{n}\right) \stackrel{\text { def }}{=}\left(v_{12}, v_{13}, \ldots, v_{1 n}\right)
$$

is a solution of the system of differential equations

$$
Y_{1}^{\prime}=B_{1} Y_{1}
$$

whose dimension is $n-1$.

By utilizing one solution $U=\left(u_{1}, \ldots, u_{n}\right)$ of the previously known $m+1$ meromorphic vector-solutions of the system (1), (2) we decreased the dimension of this system by 1 and obtained the system (47), (44) that has $m$ meromorphic vector-solutions (46). Let $Y_{11}, Y_{12}, \ldots, Y_{1 m}$ be meromorphic vector-solutions of the system (47), (44) obtained in the described above way ( $Y_{1}$ is one of these solutions). Since $m+1$ meromorphic vector-solutions $W_{0}=U=\left(u_{1}, \ldots, u_{n}\right), W_{j}=\left(w_{j 1}, \ldots, w_{j n}\right), u_{1}, w_{j 1} \not \equiv 0, j=1, \cdots, m$ of the system $(1),(2)$ are linearly independent, we obtain that $m$ meromorphic vector-solutions $Y_{11}, Y_{12}, \ldots, Y_{1 m}$ of the system $(47),(44)$ are also linearly independent $\left(Y_{1}=\left(v_{12}, v_{13}, \ldots, v_{1 n}\right), v_{12} \not \equiv 0\right)$. From (42), (46), (10), (7) we obtain

$$
T\left(r, Y_{1}\right) \stackrel{\text { def }}{=} \max _{j=2,3, \ldots, n} T\left(r, v_{j}\right) \stackrel{(42),(7)}{\leqslant} \sum_{\substack{0 \leqslant i \leqslant m, 1 \leqslant j \leqslant n}} T\left(r, w_{i, j}\right)+O(1) .
$$

Then $W_{i}=\left(w_{i 1}, \ldots, w_{i n}\right), T\left(r, W_{i}\right) \stackrel{(10)}{=} \max _{j=1, \ldots, n} T\left(r, w_{i j}\right) ; i=0,1, \ldots, m$;

$$
\begin{gathered}
\sum_{j=1}^{n} T\left(r, w_{i, j}\right) \leqslant n \max _{j=1, \ldots, n} T\left(r, w_{i, j}\right)=n T\left(r, W_{i}\right) ; \\
\sum_{i=0}^{m} \sum_{j=1}^{n} T\left(r, w_{i, j}\right) \stackrel{(49)}{\leqslant} \sum_{i=0}^{m} n T\left(r, W_{i}\right) \leqslant n(m+1) \max _{i=0,1, \ldots, m} T\left(r, W_{i}\right) .
\end{gathered}
$$

Thus from (48) it follows

$$
\max T\left(r, Y_{1}\right) \stackrel{\text { def }}{=} \max _{t=1, \ldots, m} T\left(r, Y_{1, t}\right)=O\left(\max _{i=0,1, \ldots, m} T\left(r, W_{i}\right)\right) .
$$

Under transformation (42) $m+1$ linearly-independent meromorphic vector-solutions $W_{k}(z)$, $k=0,1, \ldots, m$ of the system (1), (2) become $m$ linearly-independent meromorphic vectorsolutions $Y_{11}, Y_{12}, \ldots, Y_{1 m}$ of the form (46) of the system (47), (44) for which the estimate (50) is valid. 
By using solutions $Y_{11}, Y_{12}, \ldots, Y_{1 m}$ let us decrease the dimension of the matrix $A$ another $m-1$ times and receive the systems of differential equations

$$
Y_{k}^{\prime}=B_{k} Y_{k}, \quad k=1,2, \ldots, m,
$$

of the dimension $n-k$ where $Y_{k}=\left(v_{k, k+1}, v_{k, k+2}, \ldots, v_{k, n}\right)$ and the matrix

$$
B_{k}=\left(\begin{array}{ccccc}
s_{k+1}-p_{k} v_{k-1, k+1} / v_{k-1, k} & p_{k+1} & 0 & \ldots & 0 \\
a_{k+2, k+1}-p_{k} v_{k-1, k+2} / v_{k-1, k} & s_{k+2} & p_{k+2} & \ldots & 0 \\
\ldots \ldots \ldots \ldots \ldots \ldots \ldots \ldots \ldots \ldots \ldots & \ldots \ldots \ldots \ldots \ldots & \ldots \ldots & \ldots \ldots \\
a_{n, k+1}-p_{k} v_{k-1, n} / v_{k-1, k} & a_{n, k+2} & a_{n, k+3} & \ldots & s_{n}
\end{array}\right) .
$$

By applying the estimate (50) of the meromorphic vector-solution of the system (51) several times $(k=1, \ldots, m)$ we finally obtain

$$
\max T\left(r, Y_{k}\right) \stackrel{\text { def }}{=} \max _{t=1,2, \ldots, m-k+1} T\left(r, Y_{k, t}\right)=O\left(\max _{i=0,1, \ldots, m} T\left(r, W_{i}\right)\right) .
$$

For the meromorphic solution $Y_{k}=\left(v_{k, k+1}, v_{k, k+2}, \ldots, v_{k, n}\right)$ of the system (51), (52) let us put into the correspondence the vector $h_{k}=\left(h_{k, k+1}, h_{k, k+2}, \ldots, h_{k, n}\right)$, where $h_{k, k+p}=$ $v_{k, k+p}^{\prime} / v_{k, k+p}, p=1,2, \ldots, n-k ; k=1,2, \ldots, m$. Then

$$
\begin{gathered}
m\left(r, h_{k, k+p}\right)=m\left(r, \frac{v_{k, k+p}^{\prime}}{v_{k, k+p}}\right) \stackrel{(6)}{=} O\left(\ln ^{+} T\left(r, v_{k, k+p}\right)+\ln r\right) \stackrel{(10)}{=} \\
=O\left(\ln ^{+}\left(\max _{t=1,2, \ldots, m-k+1} T\left(r, Y_{k, t}\right)\right)+\ln r\right) \stackrel{(53)}{=} \\
=O\left(\ln ^{+}\left(\max _{i=0,1, \ldots, m} T\left(r, W_{i}\right)\right)+\ln r\right), p=1, \ldots, n-k ; k=1, \ldots, m,
\end{gathered}
$$

$r \notin E$. We will use the following lemma.

Lemma 3. The following equality holds true $(j \in \mathbb{N}, j \leqslant n-m)$

$$
d_{j 1}\left(B_{m}\right)=d_{j, m+1}(A)+d_{j, m}(A)+\ldots+d_{j, 1}(A)+\tilde{P}_{m j},
$$

$\tilde{P}_{m j}=\tilde{P}_{m j}\left(h_{k, k+p}, d_{\nu, s}(A)\right)$ are polynomials in $h_{k, k+p}, k=0,1, \ldots, m-1 ; \quad p=1,2, \ldots, j$, and $d_{\nu, s}(A), s=1,2, \ldots, m+j ; \nu \leqslant j-1$, of degree no more than 1 in every function $h_{k, k+p}, d_{\nu, s}(A)$.

Let us continue the proof of the theorem. By decreasing the dimension of the matrix $A$ we used $m$ meromorphic vector-solutions. Since we have assumed that there are $m+1$ such solutions of system (1), (2) then the system $Y_{m}^{\prime}=B_{m} Y_{m}$ (see (51), (52)) has at least one more non-trivial meromorphic vector-solution $Y_{m}=\left(v_{m, m+1}, v_{m, m+2}, \ldots, v_{m, n}\right)$ for which (see (53)) the following estimate holds

$$
T\left(r, Y_{m}\right)=O\left(\max _{i=0,1, \ldots, m} T\left(r, W_{i}\right)\right) .
$$

By transforming the system $Y_{m}^{\prime}=B_{m} Y_{m}$ to the form similar to (36) we get the system of linear homogeneous equations with the matrix $Q_{n-m}\left(B_{m}, h_{m}\right)$ (see $(24)$ ) with the nontrivial solution $Y_{m}=\left(v_{m, m+1}, v_{m, m+2}, \ldots, v_{m, n}\right)$. Thus $Q_{n-m}\left(B_{m}, h_{m}\right) \equiv 0$. Hence, taking into account (25), we obtain

$$
\left(h_{m}=\left(h_{m, m+1} ; h_{m, m+2} ; \ldots ; h_{m, m+i} ; \ldots ; h_{m, n}\right), h_{m, m+i}=\frac{v_{m, m+i}^{\prime}}{v_{m, m+i}},\right.
$$




$$
\begin{gathered}
\left.i=1,2, \ldots, n-m ; \quad Q_{0}\left(B_{m}, h_{m}\right)=1, d_{0, n-m+1}\left(B_{m}\right)=1\right) \\
d_{n-m, 1}\left(B_{m}\right)=h_{m, n} Q_{n-m-1}\left(B_{m}, h_{m}\right)+\sum_{i=0}^{n-m-2} Q_{i}\left(B_{m}, h_{m}\right) h_{m, m+i+1} d_{n-m-i-1, i+2}\left(B_{m}\right) .
\end{gathered}
$$

Let us apply in (58) to $Q_{i}\left(B_{m}, h_{m}\right), i \leqslant n-m-1$, Lemma 1 (see $(26)$ ). To $d_{j 1}\left(B_{m}\right), j \leqslant$ $n-m-1$, let us apply the formula (55). By taking into account that $d_{j, t}\left(B_{m}\right)=d_{j, m+t}(A)$ for $t \geqslant 2$ and $j=1,2, \ldots, n-m$ (see (52)), we obtain

$$
d_{n-m, 1}\left(B_{m}\right) \stackrel{(58)}{=} P\left(d_{\nu, s}(A), h_{k, k+p}\right)
$$

where $P$ is a polynomial of degree no more than 1 in $d_{\nu, s}(A), \nu<n-m, s=1, \ldots, n$ and $h_{k, k+p}, k=0,1, \ldots, m ; p=1, \ldots, n-m$. From (55) at $j=n-m$ it follows

$$
d_{n-m, 1}\left(B_{m}\right)=d_{n-m, m+1}(A)+d_{n-m, m}(A)+\ldots+d_{n-m, 1}(A)+\tilde{P}_{m, n-m}
$$

$\tilde{P}_{m, n-m}=\tilde{P}_{m, n-m}\left(h_{k, k+p}, d_{\nu, s}(A)\right)$ is the polynomial in $h_{k, k+p}, k=0,1, \ldots, m-1 ; p=$ $1,2, \ldots, n-m$, and $d_{\nu, s}(A), s=1,2, \ldots, n ; \nu \leqslant n-m-1$, of degree no more than 1 . By taking into account the definition of $H_{n-m}(A)(19)$ and also the equalities (59), (60) and properties of the polynomials $P\left(d_{\nu, s}(A), h_{m, m+p}\right), \tilde{P}_{m, n-m}$ we obtain

$$
H_{n-m}(A)=d_{n-m, m+1}(A)+d_{n-m, m}(A)+\ldots+d_{n-m, 1}(A)=R_{m, n-m},
$$

$R_{m, n-m}=R_{m, n-m}\left(h_{k, k+p}, d_{\nu, s}(A)\right)$ is a polynomial in $h_{k, k+p}, k=0,1, \ldots, m$;

$p=1,2, \ldots, n-m$, and $d_{\nu, s}(A), s=1,2, \ldots, n ; \nu \leqslant n-m-1$, of degree no more than 1 in every variable. From the equality (61) and by taking into account properties of the polynomials $R_{m, n-m}$ we obtain $(r \notin E)$

$$
\begin{gathered}
m\left(r, H_{n-m}(A)\right) \stackrel{(8)}{\leqslant} \sum_{\substack{k=0,1, \ldots, m, p=1, \ldots, n-m}} m\left(r, h_{k, k+p}\right)+\sum_{\substack{s=1,2, \ldots, n, \nu \leqslant n-m-1}} m\left(r, d_{\nu, s}(A)\right)+ \\
+O(1) \stackrel{(54)}{=} O\left(\ln ^{+}\left(\max _{i=0,1, \ldots, m} T\left(r, W_{i}\right)\right)+\ln r\right)+\sum_{\substack{s=1,2, \ldots, n, \nu \leqslant n-m-1}} m\left(r, d_{\nu, s}(A)\right) .
\end{gathered}
$$

From (20) we have

$$
\beta_{\nu s} \stackrel{\text { def }}{=} \sigma_{\varphi}^{0}\left[d_{\nu s}\right]<\sigma_{\varphi}^{0}\left[H_{n-m}(A)\right] \stackrel{\text { def }}{=} \alpha ; \quad \max \beta_{\nu s} \stackrel{\text { def }}{=} \beta<\alpha,
$$

$s=1, \ldots, n ; \quad \nu=1, \ldots, n-m+1$. Similar to $(40)$ we obtain

$$
m\left(r, d_{\nu s}\right)<\ln \varphi^{-1}\left(\ln r^{\beta+\varepsilon}\right), \quad \varepsilon>0 .
$$

Let us denote

$$
\sigma_{i}=\sigma_{\varphi}^{1}\left[W_{i}\right], \quad i=0,1, \ldots, m ; \quad \sigma=\max \sigma_{i} \stackrel{(21)}{<} \sigma_{\varphi}^{1}\left[H_{n-m}(A)\right] .
$$

Then by taking into account (16) we obtain

$$
T\left(r, W_{i}\right)<\varphi^{-1}\left(\ln r^{\sigma_{i}+\varepsilon}\right) \leqslant \varphi^{-1}\left(\ln r^{\sigma+\varepsilon}\right), \quad \varepsilon>0, \quad r>r_{0} .
$$


From $(14),(62),(64),(66)$ it follows $(K=$ const $>0)$

$$
m\left(r, H_{n-m}(A)\right)<K\left(\ln \varphi^{-1}\left(\ln r^{\sigma+\varepsilon}\right)+\ln \varphi^{-1}\left(\ln r^{\beta+\varepsilon}\right)\right)<2 K \ln \varphi^{-1}\left(\ln r^{\max (\sigma, \beta)+\varepsilon}\right), \quad r \notin E .
$$

Similar to (33) we obtain

$$
m\left(r, H_{n-m}(A)\right)=O\left(\ln \varphi^{-1}\left(\ln r^{\max (\sigma, \beta)+2 \varepsilon}\right)\right), r>r_{0}, \quad \varepsilon>0 .
$$

Thus

$$
\begin{gathered}
\varphi\left(e^{m\left(r, H_{n-m}(A)\right)}\right)=\varphi\left(e^{O\left(\ln \varphi^{-1}\left(\ln r^{\max (\sigma, \beta)+2 \varepsilon}\right)\right)}\right) \stackrel{(13)}{<} \\
<(1+o(1)) \varphi\left(e^{\ln \varphi^{-1}\left(\ln r^{\max (\sigma, \beta)+2 \varepsilon}\right)}\right)=(1+o(1)) \ln r^{\max (\sigma, \beta)+2 \varepsilon} .
\end{gathered}
$$

From here and from $(12)$ we have $\sigma_{\varphi}^{0}\left[H_{n-m}(A)\right] \leqslant \max (\sigma, \beta)$, which contradicts (63), (65).

The case where in (2) some of $p_{j} \equiv 0$, shall be considered in a way similar to [2]. The proof of Theorem 2 is similar to that of Theorem 1.

Proof of Lemma 3. By taking into account (45) let us represent $d_{j 1}\left(B_{m}\right)$ via the determinants of the matrix $B_{m-1}\left(B_{0}=A, d_{0, j+2}\left(B_{m-1}\right)=1\right.$ (see (19), (2)))

$$
d_{j 1}\left(B_{m}\right)=d_{j 2}\left(B_{m-1}\right)+Q_{j}\left(B_{m-1}, h_{m-1}\right)+\sum_{i=1}^{j-1} Q_{i}\left(B_{m-1}, h_{m-1}\right) d_{j-i, i+2}\left(B_{m-1}\right) .
$$

By using (24) we have $Q_{0}(A, h) \equiv 1, Q_{1}(A, h)=s_{1}-h_{1}=d_{11}(A)-h_{1}$,

$$
Q_{0}\left(B_{m-1}, h_{m-1}\right) \equiv 1, \quad Q_{1}\left(B_{m-1}, h_{m-1}\right)=d_{11}\left(B_{m-1}\right)-h_{m-1, m},
$$

where $h_{m-1}=\left(h_{m-1, m} ; h_{m-1, m+1} ; \ldots ; h_{m-1, n}\right), h_{m-1, m+i}=\frac{v_{m-1, m+i}^{\prime}}{v_{m-1, m+i}}, i=0,1, \ldots, n-m$. Thus $\left(d_{0, j+1}\left(B_{m-1}\right)=1\right)$

$$
\begin{gathered}
Q_{j}\left(B_{m-1}, h_{m-1}\right) \stackrel{(25)}{=} d_{j, 1}\left(B_{m-1}\right)-h_{m-1, m} d_{j-1,2}\left(B_{m-1}\right)- \\
-\left(d_{11}\left(B_{m-1}\right)-h_{m-1, m}\right) h_{m-1, m+1} d_{j-2,3}\left(B_{m-1}\right)- \\
-\sum_{i=2}^{j-1} Q_{i}\left(B_{m-1}, h_{m-1}\right) h_{m-1, m+i} d_{j-i-1, i+2}\left(B_{m-1}\right) \stackrel{(26)}{=} \\
=d_{j, 1}-h_{m-1, m} d_{j-1,2}-\left(d_{11}-h_{m-1, m}\right) h_{m-1, m+1} d_{j-2,3}- \\
-\sum_{i=2}^{j-1}\left(d_{i 1}-d_{i-1,1} h_{m-1, m-1+i}+\sum_{t=0}^{i-2} d_{t 1} P_{t i}\right) h_{m-1, m+i} d_{j-i-1, i+2},
\end{gathered}
$$

where $d_{t 1}=d_{t 1}\left(B_{m-1}\right) ; P_{t i}=P_{t i}\left(h_{m-1, m-1+p}, d_{\nu, s}\left(B_{m-1}\right)\right)$ are some polynomials in $h_{m-1, m-1+p}$ and $d_{\nu, s}\left(B_{m-1}\right) ; p=t+1, t+2, \ldots, i ; \quad s=t+2, t+3, \ldots, i ; \quad \nu \leqslant i-1 ; i=2,3, \ldots, j-1 ; t=$ $0,1, \ldots, i-2$ of degree no more than 1 in every $h_{m-1, m-1+p}$ and $d_{\nu, s}\left(B_{m-1}\right)$. By grouping in (68) the summands that contain $d_{i 1}=d_{i 1}\left(B_{m-1}\right), i=0,1, \ldots, j-1$, we obtain $\left(d_{0 i}=1\right)$

$$
Q_{j}\left(B_{m-1}, h_{m-1}\right)=d_{j, 1}\left(B_{m-1}\right)+\sum_{i=0}^{j-1} d_{i 1}\left(B_{m-1}\right) P_{i j}^{*}\left(h_{m-1, m-1+p}, d_{\nu, s}\left(B_{m-1}\right)\right),
$$

$P_{i j}^{*}\left(h_{m-1, m-1+p}, d_{\nu, s}\left(B_{m-1}\right)\right)$ are polynomials in $h_{m-1, m-1+p}$ and $d_{\nu, s}\left(B_{m-1}\right) ; p=i+1, i+$ $2, \ldots, j ; s=i+2, i+3, \ldots, j ; \nu \leqslant j-1 ; i=0,1, \ldots, j-1$, of degree no more than 1 on 
every of $h_{m-1, m-1+p}$ and $d_{\nu, s}\left(B_{m-1}\right)$. By transforming the sum in the right hand side of (67) $\left(Q_{1}\left(B_{m-1}, h_{m-1}\right)=d_{11}-h_{m-1, m}\right)$ :

$$
\begin{gathered}
\sum_{i=1}^{j-1} Q_{i}\left(B_{m-1}, h_{m-1}\right) d_{j-i, i+2}\left(B_{m-1}\right) \stackrel{(26)}{=}\left(d_{11}-h_{m-1, m}\right) d_{j-1,3}+ \\
+\sum_{i=2}^{j-1}\left(d_{i 1}-d_{i-1,1} h_{m-1, m-1+i}+\sum_{t=0}^{i-2} d_{t 1} P_{t i}\right) d_{j-i, i+2}= \\
=\sum_{i=0}^{j-1} d_{i 1}\left(B_{m-1}\right) P_{i j}^{\star}\left(h_{m-1, m-1+p}, d_{\nu, s}\left(B_{m-1}\right)\right), \quad d_{01}\left(B_{m-1}\right)=1,
\end{gathered}
$$

where the polynomials $P_{t i}=P_{t i}\left(h_{m-1, m-1+p}, d_{\nu, s}\left(B_{m-1}\right)\right)$ are the same as in (68); $P_{i j}^{\star}\left(h_{m-1, m-1+p}, d_{\nu, s}\left(B_{m-1}\right)\right)$ are polynomials in $h_{m-1, m-1+p}$ and $d_{\nu, s}$, of degree no more than 1 in every $h_{m-1, m-1+p}$ and $d_{\nu, s}\left(B_{m-1}\right) ; p=i+1, i+2, \ldots, j ; s=i+2, i+3, \ldots, j+1$; $\nu \leqslant j-1 ; i=1, \ldots, j-1$. By substituting (69), (70) into (67) and then grouping the summands with $d_{i 1}\left(B_{m-1}\right)$, we obtain

$$
d_{j 1}\left(B_{m}\right)=d_{j, 1}\left(B_{m-1}\right)+d_{j 2}\left(B_{m-1}\right)+\sum_{i=0}^{j-1} d_{i 1}\left(B_{m-1}\right) P_{i j}
$$

$P_{i j}=P_{i j}\left(h_{m-1, m-1+p}, d_{\nu, s}\left(B_{m-1}\right)\right)$ are polynomial in $h_{m-1, m-1+p}$ and $d_{\nu, s}\left(B_{m-1}\right)$, of degree no more than 1 in every $h_{m-1, m-1+p}$ and $d_{\nu, s}\left(B_{m-1}\right) ; \quad p=i+1, i+2, \ldots, j ; \quad s=i+2$, $i+3, \ldots, j+1 ; \quad \nu \leqslant j-1 ; i=1,2, \ldots, j-1$. But (see (52), (44), (18)) $d_{\nu, s}\left(B_{m-1}\right)=$ $d_{\nu, m+s-1}(A)$ at $s \geqslant 2$. Thus

$$
d_{j 1}\left(B_{m}\right)=d_{j, 1}\left(B_{m-1}\right)+d_{j, m+1}(A)+\sum_{i=0}^{j-1} d_{i 1}\left(B_{m-1}\right) P_{i j}\left(h_{m-1, m-1+p}, d_{\nu, m+s-1}\right),
$$

$P_{i j}\left(h_{m-1, m-1+p}, d_{\nu, m+s-1}(A)\right)$ are polynomials in $h_{m-1, m-1+p}$ and $d_{\nu, m+s-1}(A)$, of degree no more than 1 on every $h_{m-1, m-1+p}$ and $d_{\nu, m+s-1}(A) ; \quad p=i+1, i+2, \ldots, j ; \quad s=i+2$, $i+3, \ldots, j+1 ; \quad \nu \leqslant j-1 ; i=1,2, \ldots, j-1$.

Let us prove the formula (55). If $m=1$ then from (72) it follows ( $B_{0}=A, \quad h_{0}=$ $\left.\left(w_{01}^{\prime} / w_{01}, \ldots, w_{0 n}^{\prime} / w_{0 n}\right), h_{0, p}=w_{0 p}^{\prime} / w_{0 p}(\operatorname{see}(45))\right)$

$$
\begin{gathered}
d_{i 1}\left(B_{1}\right)=d_{i, 1}(A)+d_{i, 2}(A)+\sum_{t=0}^{i-1} d_{t 1}(A) P_{t i}\left(h_{0, p} ; d_{\nu, s}(A)\right)= \\
=d_{i, 1}(A)+d_{i, 2}(A)+\tilde{P}_{1 i}, \quad i \in \mathbb{N}, \quad i \leqslant n-1,
\end{gathered}
$$

$\tilde{P}_{1 i}$ is a polynomial in $h_{0, p}$ and $d_{\nu, s}(A), \quad p=1,2, \ldots, i ; \quad s=1,2, \ldots, i+1 ; \quad \nu<i$ of degree no more than 1 in every of the functions.

Let for every $i \in \mathbb{N}, i \leqslant j \leqslant n-m, 2 \leqslant m$ the following equality take place

$$
d_{i 1}\left(B_{m-1}\right)=d_{i, 1}(A)+d_{i, 2}(A)+\ldots+d_{i, m}(A)+\tilde{P}_{m-1, i}, \quad i \leqslant n-m,
$$

$\tilde{P}_{m-1, i}$ is a polynomial in $h_{0 p}, h_{1, p+1}, \ldots, h_{m-2, m-2+p}$ and $d_{\nu, s}(A) ; p=1,2, \ldots, i ; s=1,2$, $\ldots, i+m-1 ; \nu<i$ of degree no more than 1 in every of $h_{k, k+t}$ and $d_{\nu, s}(A)$. By substituting (74) into (72) we obtain

$$
\begin{gathered}
d_{j 1}\left(B_{m}\right)=d_{j, 1}(A)+d_{j, 2}(A)+\ldots+d_{j, m}(A)+d_{j, m+1}(A)+\tilde{P}_{m-1, j}+ \\
+\sum_{i=0}^{j-1}\left(d_{i, 1}(A)+\ldots+d_{i, m}(A)+\tilde{P}_{m-1, i}\right) P_{i j}\left(h_{m-1, m-1+p}, d_{\nu, m+s-1}(A)\right)= \\
=d_{j, 1}(A)+d_{j, 2}(A)+\ldots+d_{j, m+1}(A)+\tilde{P}_{m, j}
\end{gathered}
$$


$\tilde{P}_{m, j}$ is a polynomial in $h_{0 p}, h_{1, p+1}, \ldots, h_{m-1, m-1+p}$ and $d_{\nu, s}(A) ; p=1,2, \ldots, j ; s=1,2, \ldots$, $j+m ; \nu<j$ of degree no more than 1 on every $h_{k, k+t}$ and $d_{\nu, s}(A)$. Here we took into account that $\tilde{P}_{m-1, i}$ contains $d_{\nu, s}(A)$ with indices $s=1,2, \ldots, i+m-1$ and $P_{i j}\left(h_{m-1, m-1+p}\right.$, $\left.d_{\nu, m+s-1}(A)\right)$ contain $d_{\nu, m+s-1}(A)$ with indexes $s=i+2, i+3, \ldots, j+1$. Then $\tilde{P}_{m-1, i}$ includes also $h_{0 p}, h_{1, p+1}, \ldots, h_{m-2, m-2+p}$ at $p=1,2, \ldots, i$ and $P_{i j}\left(h_{m-1, m-1+p}, d_{\nu, m+s-1}(A)\right)$ contain $h_{m-1, m-1+p}$ with indices $p=i+1, i+2, \ldots, j$.

\section{REFERENCES}

1. Golubev V.V. Lectures on analytic theoury of differential equations. - Gostehizdat, Moscow; Leningrad, 1950. - 436 p. (in Russian)

2. Hengartner W. Über die Wachstumsordnung eines linearen Systems von Differentialgleichungen mit ganzen Funktionen als Koeffizienten// Comment. Math. Helv. - 1967. - V.42, №1. - P.60-80.

3. Steinmetz N. Nevanlinna Theory, Normal Families, and Algebraic Differential Equations. - Springer International Publishing AG, 2017 - 235p.

4. Chyzhykov I.E., Semochko N.S. Fast growing entire solutions of linear differential equations// Mat. Visnuk NTSh. - 2016. - V.13. - P. 68-83.

5. Mokhonko A.A. Malmquist Theorem for solutions of differential equations in the neigbourhood of logarithmic singularity// Ukrainian Math. J. - 2004. - V.56, №4. - P. 476-483. (in Ukrainain)

6. Goldberg A.A., Ostrovskii I.V., Value distribution of meromorphic functions. Translations of Mathematical Monographs, V.236, Springer, Berlin, 2008.

7. A.Z. Mokhonko, V.D. Mokhonko, Estimates for the Nevanlinna characteristics of some classes of meromorphic functions and their applications to differential equations// Sib. Math. J. - 1974. - V.15. P. 921-934. (in Russian)

8. Mokhonko A.Z. Field of algebroid functions and estimates of their Nevanlinna's characteristics// Siberian Math. J. - 1981. - V.3. - P. 213-218. (in Russian)

9. Schonhage A. Uber das Wachstum der zusammengesetzen Funktionen// Math. Z. - 1960. - V.73. P. 22-44.

10. Juneja O.P., Kapoor G.P., Bajpai S.K. On the $[p, q]$-order and lower $[p, q]$-order of an entire function// J. Reine Angev. Math. - 1976. - V.282. - P. 53-67.

11. Sheremeta M.N. About connection between absolute value of the module of entire function and absolute values of the coefficients of its series expansion// Izvestia Vuzov Mathematica. - 1967. - V.2. - P. 100108. (in Russian)

12. Bandura A., Skaskiv O., Filevych P. Properties of entire solutions of some linear PDE's// J. Appl. Math. Comput. Mech. - 2017. - V.16., №2, P. 17-28.

13. W.K. Hayman, Meromorphic function, Mir, Moscow, 1966, 287p. (in Russian)

Lviv Polytechnic National University, Lviv, Ukraine mohon@soft-hitech.com 